\title{
A Network-Layer Soft Handoff Approach for Mobile Wireless IP-Based Systems
}

\author{
Bechir Hamdaoui and Parameswaran Ramanathan
}

\begin{abstract}
Handoff is the process during which a mobile node (MN) needs to change its connectivity point to the wireless internetwork from one access node (AN) to another during an ongoing communication. If MNs are allowed to have two or more simultaneous connections to the internetwork through different ANs, then the handoff is said to be soft; otherwise, it is said to be hard. Traditionally, during forward-link soft handoff, multiple identical copies of each packet are simultaneously transmitted to the MN through the associated ANs. At the MN's physical-layer, the received signals are combined on a bit-by-bit basis resulting in improving the bit-error rate. However, this approach requires tight synchronizatin of the ANs involved in the soft handoff. In addition, as shown in the literature, the capacity often decreases due to the increase of the number of channels used by MNs during soft handoff.

In this paper, we propose, analyze, simulate, and implement a soft handoff scheme called soft handoff over IP (SHIP) for forward-link that 1) overcomes the need for synchronization and 2) increases the capacity of the network. Through both analytic and simulation studies, we show that SHIP achieves significant performance improvements. We derive analytic expressions of the powercapacity relationship for two-dimensional (2-D) and one-dimensional (1-D) cell models. By comparing our scheme with the hard handoff, we empirically show that the capacity increases by about $30 \%$ and $20 \%$, respectively, for the 2-D and 1-D cell models. Further, the simulation results show that SHIP saves up to $30 \%$ of the total power consumed by the ANs.
\end{abstract}

Index Terms-Hard handoff, mobile IP networks, multiple descriptions, network capacity, packet error rate (PER), soft handoff, wireless systems.

\section{INTRODUCTION}

$\mathbf{I}^{\mathrm{N}}$ $\mathrm{N}$ RECENT YEARS, there has been a growing demand for mobile computers such as laptops and hand-held devices. Mobile computers, referred to here as mobile nodes (MNs), often need to continuously access the wireless internetwork (e.g., Internet) without losing their ability to communicate. However, during an ongoing wireless communication, a $\mathrm{MN}$ is very likely to experience a handoff process during which the $\mathrm{MN}$ needs to change its connectivity point to the network from one wireless access node (AN) to another. A handoff is said to be hard when MNs can communicate with only one AN at all

\footnotetext{
Manuscript received February 1, 2003; revised October 7, 2003. This work was supported in part by U.S. Army Research under Grant DAAD19-01-1-0504 and in part from the Pennsylvania State University under a Subrecipient Agreement S01-24 . Any opinions, findings, and conclusions or recommendations expressed in this publication are those of the author(s) and do not necessarily reflect the views of the U.S. Army Research Office.

The authors are with the Department of Electrical and Computer Engineering, University of Wisconsin, Madison, WI 53706 USA (e-mail: hamdaoui@cae.wisc.edu; parmesh@ece.wisc.edu).

Digital Object Identifier 10.1109/JSAC.2004.825986
}

times; that is, in a hard handoff, the switching from one AN to another occurs instantaneously. On the other hand, a handoff is said to be soft when MNs are allowed to have two or more simultaneous connections to the network through different ANs. Soft handoff is typically allowed when the MN enters the boundary region of the coverage area of the associated ANs, which results in a smooth transition of the MN across the ANs.

For reverse-link communication, each packet transmitted by a MN is received, demodulated, and decoded by all ANs involved in the soft handoff. The most likely correct packet is then selected for forwarding to the destination. In [1] and [6], the authors show that on the reverse-link the network does not lose capacity due to soft handoff. This is because no extra channels are required to perform the soft handoff.

During forward-link soft handoff process, multiple identical copies of each packet are simultaneously transmitted to the MN through different ANs. The MN then combines the received signals on a bit-by-bit basis resulting in a stronger signal-to-interference and noise ratio (SINR), which in turn reduces the bit-error rate (BER). This approach requires all ANs to be tightly synchronized and a scheduling scheme which ensures that ANs involved in the soft handoff will transmit the same packet [7]. Such a synchronized scheduling is difficult to achieve. Also, since this approach requires each AN to allocate a separate channel, there is a decrease in the capacity of the network [1]-[5], [8], [9].

In this paper, we propose a soft handoff approach for forward-link that 1) overcomes the need for synchronization and 2) increases the capacity of the network. The idea is to combine the information received from different ANs at the network-layer instead of the physical-layer. The MN's network-layer gets a copy of the packet from each of the ANs, and it constructs one packet to forward to the upper-layer. This feature eliminates the need for tight synchronization since the network-layer allows more flexible delay than that allowed at the physical-layer. The proposed soft handoff scheme also exploits the repetition of the packet; that is, instead of sending multiple identical copies, the scheme tunnels multiple different copies, called multiple descriptions, for each packet. Using multiple descriptions results in improving the quality of transmissions by reducing the BER of the channels [10]-[12], or increasing the capacity of the network in exchange for the channel-quality improvement.

We derive analytic expressions to characterize the power-capacity relationship of the network for both the two-dimensional (2-D) and the one-dimensional (1-D) cell models under the hard and soft handoff schemes. We show that the proposed scheme performs substantially better than 


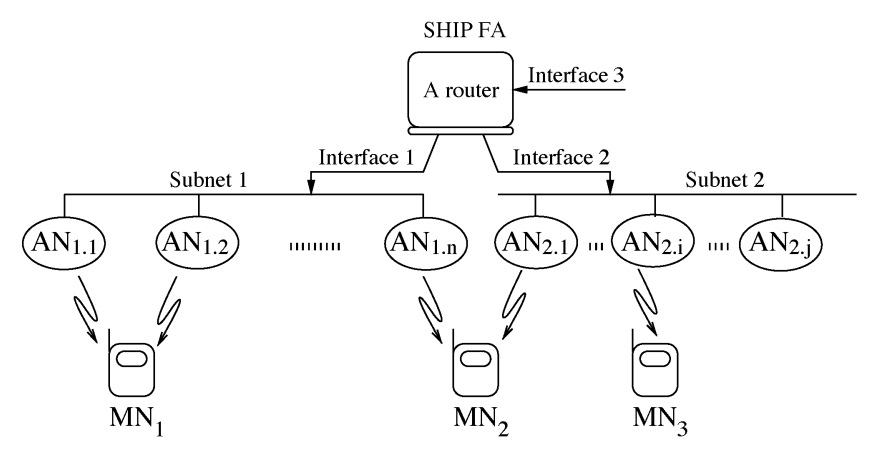

Fig. 1. SHIP functional entities.

the repetition scheme, where the descriptions are identical. By comparing our proposed scheme with the hard handoff scheme, we show that the capacity of the network in a forward-link traffic is increased by about $30 \%$ and $20 \%$, respectively, for the 2-D and 1-D cell models. A comparison of the capacity gain/loss of the proposed and repetition schemes is also studied for other parameters of the network. Through simulation studies, we also show that the proposed scheme saves up to $30 \%$ and $20 \%$ of the total consumed power when compared with the hard handoff scheme, respectively, for the 2-D and 1-D cell models.

The proposed scheme is implemented in the network stack of the Linux Kernel v2.4.18-3 from Red Hat. We demonstrate, through the experimental testbed, the ease of implementation of the proposed technique.

The rest of the paper is organized as follows. Section II presents the functionality and the design of the proposed approach. Section III describes the system model used in this research work to evaluate the proposed scheme. In Section IV, we analytically evaluate the capacity-power relationship of the proposed and the repetition soft handoff-based networks. In Section V, an empirical evaluation of the forward-link performance is developed. A comparison and analysis of both the proposed and the repetition schemes are also provided. Simulation results of the proposed scheme are provided in Section VI. The implementation of the proposed scheme is illustrated in Section VII. In Section VIII, we discuss the latency of SHIP. We conclude the paper in Section IX.

\section{PROPOSED APPROACH: SHIP}

In this section, we present the proposed soft handoff over Internet protocol (IP) scheme. We refer to our scheme as SHIP. For simplicity of presentation, we assume that a $\mathrm{MN}$ is connected to two ANs during soft handoff.

\section{A. Soft Handoff Over IP (SHIP)}

Fig. 1 shows a typical architecture of a wireless network. The network has two subnetworks: subnet 1 and subnet 2. Each subnetwork consists of ANs and MNs. In Fig. 1, $\mathrm{MN}_{1}$ and $\mathrm{MN}_{2}$ are under soft handoff. Traditionally, for forward-link traffic, two copies of each packet are simultaneously transmitted to
$\mathrm{MN}_{1}$ through $\mathrm{AN}_{1.1}$ and $\mathrm{AN}_{1.2} . \mathrm{MN}_{1}$ combines the two received signals at the physical-layer on a bit-by-bit basis. Thus, this approach requires both ANs to be tightly synchronized. It also requires a scheduling scheme to ensure that all involved ANs transmit the same packet during soft handoff. To eliminate the need for synchronization, SHIP combines both copies of each packet at the $\mathrm{MN}_{1}$ 's network-layer, and constructs one packet for further processing at the higher layer. In addition, instead of sending two identical copies, SHIP generates two descriptions of the packet and delivers them through the ANs; i.e., $\mathrm{AN}_{1.1}$ relays one description, while the other description is relayed through $\mathrm{AN}_{1.2}$. Although many different schemes can be used for generating the two descriptions, the paper assumes that Reed-Solomon codes [13] are used for this purpose. In particular, if $K$ is the number of bits in the packet, then a $(2 K$, $K$ ) Reed-Solomon code is used to generate $K$ check bits. The $K$-bits in the original packet form the first description, while the $K$ check bits form the second description. We refer to the former as packet description and the latter as Reed-Solomon description. The network-layer of the MN uses the error correcting properties of Reed-Solomon code to construct a $K$-bit packet for forwarding to the higher layer.

Forward-link soft handoff mode is usually detected and initiated based on link-layer measurements of the strength of the received signal. There is a SINR threshold below which a particular MN is allowed to enter the soft handoff mode. The linklayer detection mechanism is beyond the scope of this work. SHIP assumes that it is the task of the MN's link-layer to inform its network-layer whenever the MN enters the soft handoff process. When the MN enters soft handoff, its network-layer informs the forwarding agent so that it starts duplicating the packets. SHIP scheme uses the following exchange of messages.

1) The MN's link-layer informs (triggers) its network-layer of the soft handoff mode.

2) The power control scheme at the ANs adjusts the powers to satisfy the soft handoff target SINRs.

3) The MN sends a SHIP-start-duplication message (SHIP-SD message) to the SHIP forwarding agent (SHIP-FA) to which the $\mathrm{MN}$ is currently registered. Upon receiving this message, SHIP-FA creates an entry in its SHIP table for the MN. As long as this entry exists, the SHIP-FA sends two different descriptions for each packet. Details concerning the SHIP table are provided later in this section. During soft handoff transmissions, descriptions of packets should have a special bit indicating such mode. We call this bit SHIP mode bit.

When the MN leaves the soft handoff zone, its link-layer triggers its network-layer notifying it of the end of the soft handoff mode. To end the soft handoff mode, SHIP uses the following messages.

1) The link-layer of the MN sends a SHIP-notify message by which the MN informs both ANs to switch to the nonhandoff regime.

2) The MN then notifies the SHIP-FA of the end of the soft handoff regime through a SHIP-end-duplication message (SHIP-ED message). Upon receiving the message, the SHIP-FA stops duplicating packets. 


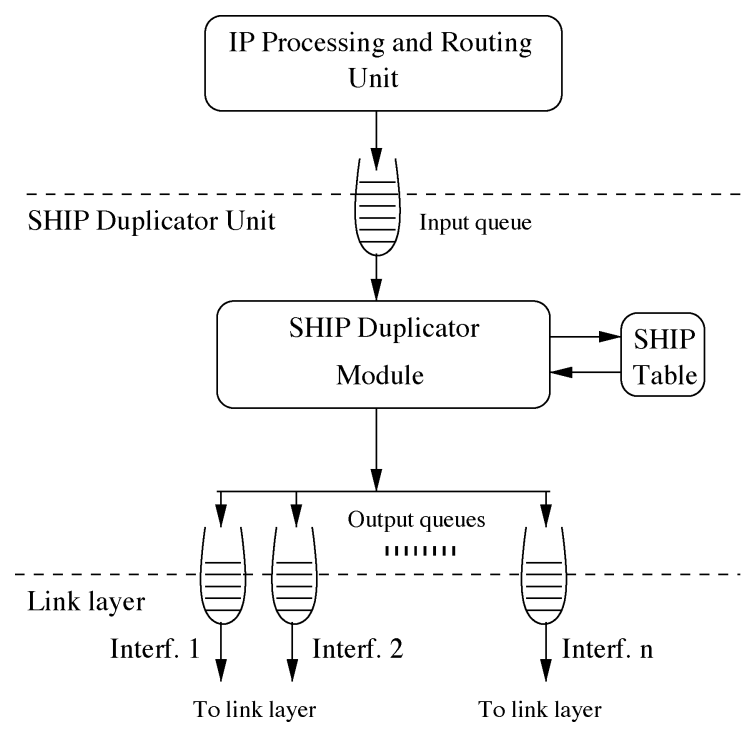

Fig. 2. SHIP-DU architecture.

\section{B. SHIP Architecture}

SHIP uses two units: SHIP-duplicator unit (SHIP-DU) and SHIP-combiner unit (SHIP-CU). The SHIP-DU runs at the SHIP-FA and is responsible for duplicating and forwarding the stream of packets of any MN experiencing a soft handoff process. The SHIP-CU is implemented only by MNs. It combines the received descriptions of each packet and forwards it to the higher layer for further processing.

1) SHIP-DU: Fig. 2 shows the architecture of the SHIP-DU. It consists of the following structures.

- SHIP Queues: SHIP-DU has two types of queues: single input queue and multiple output queues. The input queue holds packets coming from the IP processing and routing unit. SHIP-DU assumes that the IP processing and routing unit stores the physical interface and the physical address [e.g, medium access control (MAC)] along with each packet into the input queue. The output queues are used to store packets going to the link-layer for transmission. For each interface there is one output queue.

- SHIP Table: This table contains information about MNs that are under soft handoff mode. There is an entry in the table for every MN in soft handoff. Each entry of the table has five fields: MN's IP address, physical address 1, physical interface 1 , physical address 2 , and physical interface 2. The SHIP table is used by the SHIP duplicator module to map the MN's IP address into its physical addresses and interfaces, as described later. For example, referring to Fig. 1, if we assume that only $\mathrm{MN}_{1}$ and $\mathrm{MN}_{2}$ are under soft handoff, then the SHIP table looks like the table shown in Fig. 3. Note that $\mathrm{MN}_{3}$ does not have an entry in the table since it is not under soft handoff regime.

- SHIP Duplicator Module (SHIP-DM): Fig. 2 illustrates the SHIP-DM functions and its interaction with the other modules. This module is responsible for checking the handoff mode of the MN to which the packet is destined. SHIP-DM consults the SHIP table to find out whether a particular $\mathrm{MN}$ is under soft handoff or not. Along with the packet itself, the IP processing and routing unit delivers the MN's physical address and the MN's physical interface to the input queue. If the $\mathrm{MN}$ is not under soft handoff, SHIP-DM passes the packet and its information to the link-layer exactly as received. In case of soft handoff mode, SHIP-DM generates a Reed-Solomon description for the packet. It then sends the Reed-Solomon description with one pair of physical address-physical interface, and the packet description with the other pair to their corresponding output queues. The output queue is selected based on the physical interface to which the packet is intended to be sent. The physical address-physical interface pairs are looked up in the SHIP table. SHIP-DM also sets the SHIP mode bit on for both descriptions, to indicate that the packets are soft handoff packets.

SHIP-DU functions as follows.

1) Wait until the input queue is not empty, then dequeue an entry from it.

2) Extract the IP packet (and, thus, MN's IP address), the physical address, and the physical interface from the queue entry.

3) Consult the SHIP table to find out if there is an entry to the MN's IP address.

a) If entry is not found:

i) Store the packet and its related information in the output queue exactly as received.

ii) Return to step 1.

b) If entry is found (i.e., the $\mathrm{MN}$ is under soft handoff mode):

i) Generate the Reed-Solomon description of the packet.

ii) Set the SHIP mode bit on for the ReedSolomon description.

iii) Store the Reed-Solomon description along with one physical address-physical interface pair into the corresponding output queue.

iv) Set the SHIP mode bit on for the packet description.

v) Store the packet description and the second pair of physical address-physical interface into the corresponding output queue.

vi) Return to step 1.

2) SHIP-CU: This unit does the reciprocal function of the SHIP-DU. It combines the received descriptions of each packet, corrects errors if there are any, and then passes a corrected copy to the IP processing and routing unit. Fig. 4 shows the SHIP-CU architectural entities and their interaction.

- SHIP Queues: SHIP-CU also maintains two sets of queues: two input queues and one output queue. An input queue is used to store packets coming from each AN. The output queue is used to store packets leaving SHIP-CU toward the IP processing and routing unit.

- SHIP Array: This structure is used to store the first arriving description of each packet. Each entry holds the description until either the second description arrives or the SHIP timer expires. 


\begin{tabular}{|c|c|c|c|c|}
\hline MN's IP address & MN's Phy. Addr. 1 & MN's Phy. Interf. 1 & MN's Phy. Addr. 2 & MN's Phy. Interf. 2 \\
\hline \hline MN 1 & MAC of AN 1.1 & Interface 1 & MAC of AN 1.2 & Interface 1 \\
\hline MN 2 & MAC of AN 1.n & Interface 1 & MAC of AN 2.1 & Interface 2 \\
\hline$\vdots$ & $\vdots$ & $\vdots$ & $\vdots$ & $\vdots$ \\
\hline & $\vdots$ & $\vdots$ & $\vdots$ & $\vdots$ \\
\hline & & & & \\
\hline
\end{tabular}

Fig. 3. Example of the SHIP table.

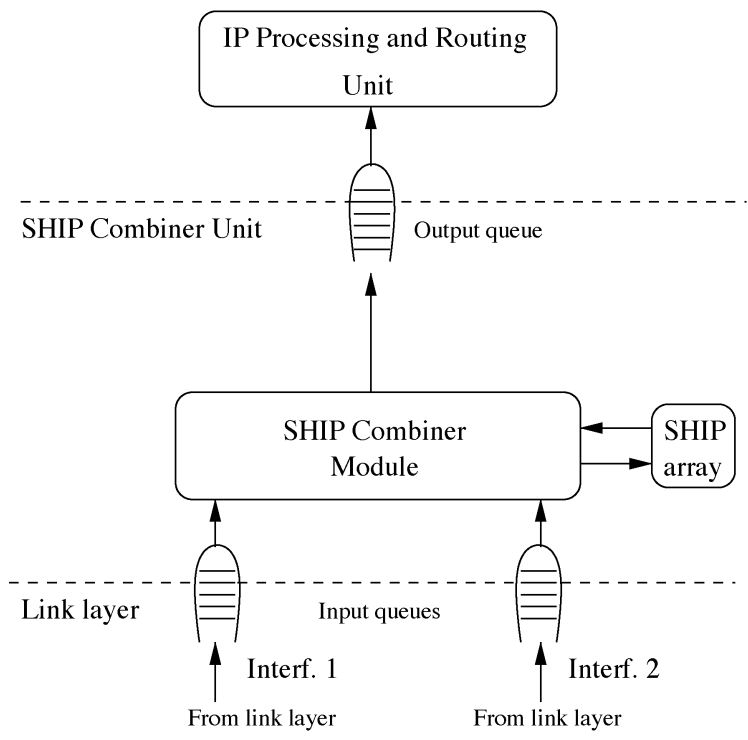

Fig. 4. SHIP-CU architecture.

- SHIP Combiner Module (SHIP-CM): This module is responsible for checking whether every received packet is a soft handoff packet. The module uses SHIP mode bit to find out about the handoff mode. If the bit is not set, then it passes the packet as received. If the bit is set on, then it waits until the second description reaches the input queue. When both descriptions are ready, SHIP-CM uses them to correct errors if possible, and generates one packet. It then enqueues the corrected packet to the output queue. SHIP-CM is also responsible for checking the SHIP timer of each SHIP array entry. Whenever the SHIP timer expires, SHIP-CM assumes that the second description is comprised of all 0 s and generates a packet using the Reed-Solomon code. SHIP-CM then frees the entry.

The SHIP-CU proceeds as follows.

1) Check the input queues.

a) If entry is found

i) Dequeue an entry.

b) Else

i) Go to step 3).

2) Check the SHIP mode bit.

a) If the SHIP mode bit is not set

i) Store the entry in the output queue exactly as received.

b) Else (i.e., $\mathrm{MN}$ is under soft handoff mode) i) Check the SHIP Array for an entry that matches the received description.

A) If entry is not found

- Create an entry in the SHIP Array and store the description into it.

- Set the SHIP timer for this new entry.

B) Else

-Retrieve the first description from the SHIP Array.

-Use the Reed-Solomon description to correct errors, if any.

- Put the combined version into the output queue.

-Free the SHIP Array entry.

3) Check the SHIP timer.

a) For all SHIP Array entries, do

i) If entry is expired

A) Create a second description filled with $0 \mathrm{~s}$.

B) Generate a packet using the Reed-Solomon code.

C) Store the packet into the output queue.

D) Free the entry.

4) Return to step 1).

\section{SYSTEM MODEL}

\section{A. Cell Model}

The wireless network is divided into regions called cells. Each cell is covered by an access point referred to as access node (AN). The ANs are the base stations in cellular networks. Each cell has MNs that communicate directly with the AN. Each MN is assumed to have an infinite number of packets to receive from the AN. Each packet has $\mathcal{L}$ symbols of $\mathcal{S}$-bits each. The cell model consists of one studied cell $k$ situated in between neighbor cells $m \in\{1,2,3, \ldots\}$. Each cell $k$ consists of two zones: nonhandoff zone $N_{k}$, and soft handoff zone $S_{k}$. When a MN enters the soft handoff zone $S_{k}$, it is simultaneously connected to multiple cells: the cell $k$ and its neighbor cells. However, as mentioned in Section II, for simplicity, we assume that a MN connects to only two cells, its cell $k$ and its nearest neighbor cell, during soft handoff. On the other hand, a $\mathrm{MN}$ can communicate only with the cell $k$ if it is located in the nonhandoff zone $N_{k}$.

Let $\mathrm{AN}_{k}$ designate the $\mathrm{AN}$ situated in cell $k$. Each $\mathrm{AN}_{k}$ is responsible for forwarding packets to all MNs located in 1) the nonhandoff zone $N_{k}$ and 2) the soft handoff zone $S_{k}$. Let $\mathrm{AN}_{k}$ mean both the cell $k$ and the AN $k$; the meaning should be clear 


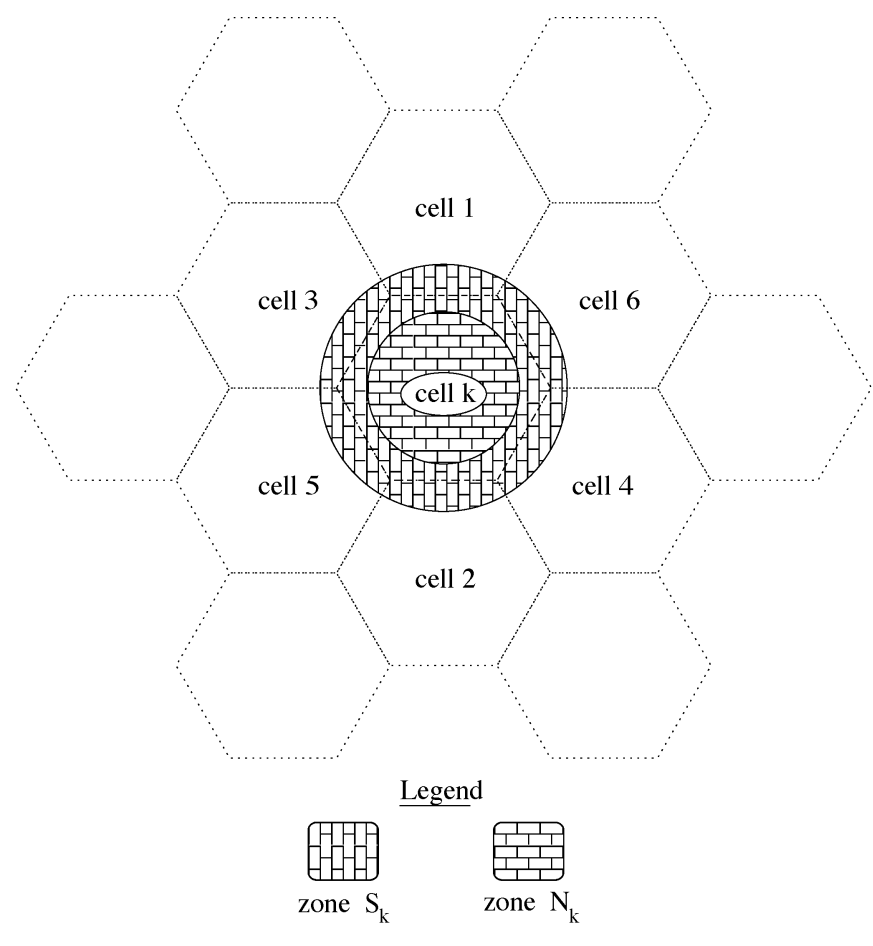

Fig. 5. Two-dimensional cell model.

from its context. Recall that the signal of a $\mathrm{MN}$ receiving from $\mathrm{AN}_{k}$ can interfere with other nearby cells. Let $\mathcal{A}_{k}$ designate the set of cells consisting of the studied cell $k$ and all its interfering neighbor cells $m \in\{1,2,3, \ldots\}$.

Practically speaking, ANs are likely to be distributed in two manners. First, ANs can be deployed in 2-D areas such as cities and towns; where MNs are spread all over the cities. In this case, ANs should cover the whole 2-D geographical area so that they service all MNs in the region. Second, unlike the first case, ANs are also likely to be deployed all along one direction. Examples of such linear model is highways where the MNs are linearly distributed along the road. Therefore, in this work, we study the two cell models: the 2-D model and the 1-D model. We refer to the 1-D model as the linear model. Fig. 5 shows the 2-D cell model where the studied cell $k$ is surrounded by its neighbor cells from all the directions. The linear cell model is shown in Fig. 6. Unlike the 2-D case, the studied cell $k$ in the linear model has neighbors only from two sides: the right and left sides.

Note that the soft handoff zone $S_{k}$ overlaps with the soft handoff zones of all the direct neighbors of cell $k$. For example, $S_{k}$ overlaps with the soft handoff zones of all cells $m, m \in$ $\{1,2,3,4,5,6\}$, in the case of the 2-D model (see Fig. 5). In the linear model, $S_{k}$ overlaps only with the soft handoff zones $S_{1}$ and $S_{2}$, respectively, of cell 1 and cell 2, as shown in Fig. 6.

\section{B. Wireless Medium Model}

The ratio of the strength of the desired signal to that of the noise and the other nearby signals is called SINR. In a forward-link communication, the SINR for a particular $\mathrm{MN}_{i}$ communicating through $\mathrm{AN}_{k}$ depends mainly on the power level $P_{i k}$ at which $\mathrm{AN}_{k}$ is transmitting to $\mathrm{MN}_{i}$, the interference level caused by other nearby transmitters, and the noise of the channel. The interference level depends on the power level at which the nearby ANs are transmitting, and the path losses between the $\mathrm{MN}_{i}$ and these interfering ANs. Let $\mathcal{H}_{i m}$ denote the path loss between $\mathrm{MN}_{i}$ and $\mathrm{AN}_{m}$. The path loss $\mathcal{H}_{i m}$ depends on the distance between the $\mathrm{MN}_{i}$ and the $\mathrm{AN}_{m}$, and the obstacles in the path between the $\mathrm{MN}_{i}$ and the $\mathrm{AN}_{m}$. We assume that $\mathcal{H}_{i m}$ depends only on the distance $l$ between $\mathrm{MN}_{i}$ and $\mathrm{AN}_{m}$. That is, $\mathcal{H}_{i m}=l^{-\alpha}$, where $\alpha \in\{1,2,3, \ldots\}$. The larger the $\alpha$, the worse the channel.

The state of a wireless medium is usually characterized by the BER, which in turn is a function of the SINR. The lower the SINR, the higher the BER, which usually means a higher packet error rate (PER). For a given MN, the PER depends on 1) the BER of the medium over which the $\mathrm{MN}$ is receiving and 2) whether the MN is under hard or soft handoff mode. Let $\Upsilon$ denote the PER of the wireless medium. Recall that for the hard handoff mode, each packet is transmitted only through one link whereas, under soft handoff mode, two descriptions are communicated for each packet. For SHIP, one of the descriptions consists of Reed-Solomon check bits. Both descriptions are identical in the repetition scheme. The PER evaluates as

$$
\Upsilon= \begin{cases}1-(1-\Phi)^{\mathcal{L S}}, & \text { for the hard handoff } \\
\left(1-(1-\Phi)^{\mathcal{L S}}\right)^{2}, & \text { for the repetition } \\
1-\sum_{i=0}^{\frac{\mathcal{L}}{2}}\left(\begin{array}{c}
2 \mathcal{L} \\
i
\end{array}\right) \Psi^{i}(1-\Psi)^{2 \mathcal{L}-i}, & \text { for SHIP }\end{cases}
$$

where $\Phi$ is the BER, $\Psi=1-(1-\Phi)^{\mathcal{S}}$ is the symbol error rate, and $\left(\begin{array}{c}2 \mathcal{L} \\ i\end{array}\right)=(2 \mathcal{L}) ! /(2 \mathcal{L}-i) ! i !$

Fig. 7 plots PER as a function of (a) the BER and (b) the SINR. From Fig. 7(b), note that to satisfy the same target PER, say $\widehat{\Upsilon}$, SHIP requires significantly less SINR as opposed to that required by using the repetition scheme. For example, to meet a target $\widehat{\Upsilon}=10 \%$, the hard handoff and the repetition soft handoff schemes require SINRs of $\approx 7$ and $\approx 5.7 \mathrm{~dB}$, respectively, whereas SHIP requires only $\approx 1.8 \mathrm{~dB}$. We also note that the lower the required PER, the bigger the gap between the required SINRs of SHIP and the repetition scheme.

\section{Power Control Model}

In a forward-link communication, the SINR for a particular $\mathrm{MN}_{i}$ receiving from $\mathrm{AN}_{k}$ depends on three factors. First, the power level received at $\mathrm{MN}_{i}$. Second, the interference level $\mathcal{I}_{i}$ seen by $\mathrm{MN}_{i}$ 's signal and caused by all other nearby transmitters, i.e., all cells belonging to $\mathcal{A}_{k}$. Third, the noise condition of the wireless medium over which the MN is communicating. We consider a white Gaussian noisy medium with variance $\sigma^{2}$.

Let $\mathrm{MN}_{i} \in \mathrm{AN}_{k}$. The SINR of $\mathrm{MN}_{i}$, also referred to as $\gamma_{i}$, can be written as

$$
\gamma_{i}=G \frac{\mathcal{H}_{i k} P_{i k}}{\mathcal{I}_{i}+G \sigma^{2}}
$$

where $G$ is the processing gain. The interference level $\mathcal{I}_{i}$ seen by a $\mathrm{MN}_{i} \in \mathrm{AN}_{k}$ is a function of the power levels at which all $\mathrm{AN}_{m}, m \in \mathcal{A}_{k}$, are transmitting, and the path losses $\mathcal{H}_{i m}$ between the $\mathrm{MN}_{i}$ and all $\mathrm{AN}_{m}, m \in \mathcal{A}_{k}$. The interference $\mathcal{I}_{i}$ can be written as

$$
\mathcal{I}_{i}=\sum_{m \in \mathcal{A}_{k}} \mathcal{I}_{i m}-\mathcal{H}_{i k} P_{i k}
$$




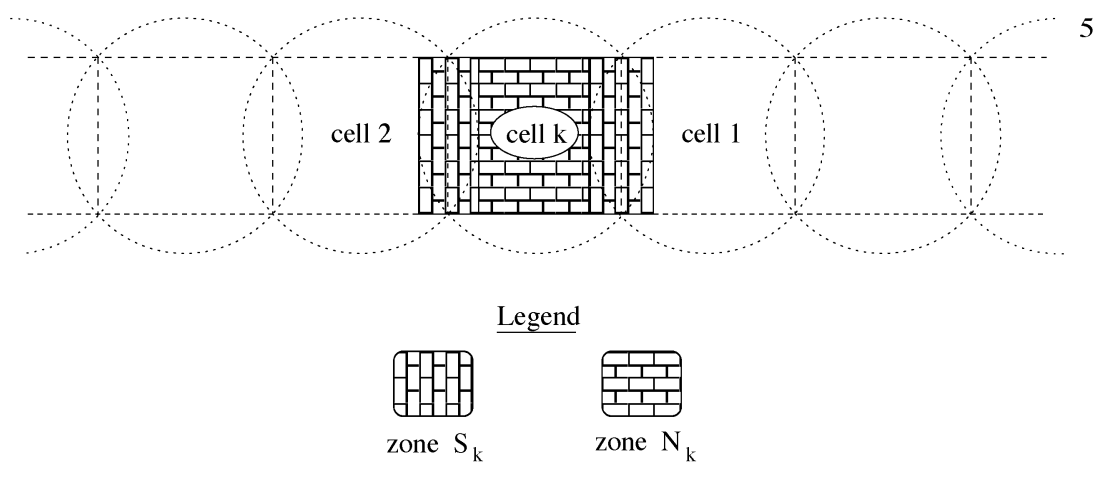

Fig. 6. Linear cell model.

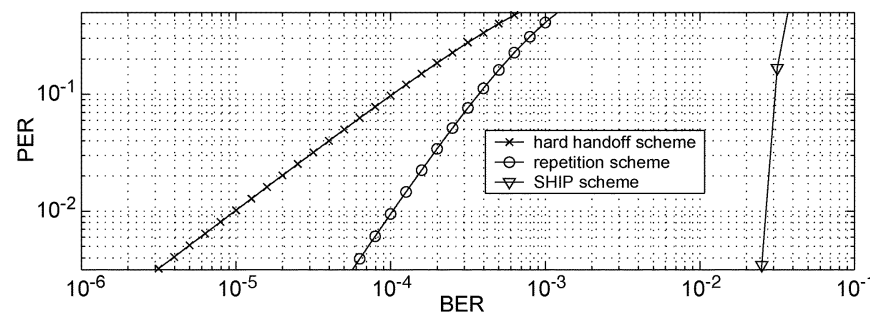

(a) $\Upsilon=f(\Phi)$



(b) $\Upsilon=f(\gamma)$.

Fig. 7. PER as function of (a) BER and (b) SINR.

where for every $m \in \mathcal{A}_{k}, \mathcal{I}_{i m}=\sum_{j \in \mathrm{AN}_{m}} \mathcal{H}_{i m} P_{j m}$ is the interference caused by $\mathrm{AN}_{m}$ and seen by $\mathrm{MN}_{i}$. Note that $\mathcal{I}_{i k}$ designates the interference caused by $\mathrm{AN}_{k}$ itself. Thus, (1) can be rewritten as

$$
\gamma_{i}=G \frac{\mathcal{H}_{i k} P_{i k}}{\sum_{m \in \mathcal{A}_{k}} \mathcal{I}_{i m}-\mathcal{H}_{i k} P_{i k}+G \sigma^{2}} .
$$

Typically, the power control scheme aims at the following. For every $\mathrm{MN}_{i} \in \mathrm{AN}_{k}$, find the power $P_{i k}$ minimizing $\sum_{j \in \mathrm{AN}_{k}} P_{j k}$ such that

$$
\begin{cases}P_{i k}>0, & \text { for all } \mathrm{MN}_{i} \\ \gamma_{i} \geq \hat{\gamma}_{i}^{N}, & \text { for } \mathrm{MN}_{i} \in N_{k} \\ \gamma_{i} \geq \hat{\gamma}_{i}^{S}, & \text { for } \mathrm{MN}_{i} \in S_{k}\end{cases}
$$

where $\gamma_{i}$ is given by (2), and $\hat{\gamma}_{i}^{N}$ and $\hat{\gamma}_{i}^{S}$ are the target SINRs of $\mathrm{MN}_{i}$ communicating from zones $N_{k}$ and $S_{k}$, respectively. These targets depend on the target PER, which in turn reflects the target quality of the communication.

\section{ANalytical Evaluation of SHIP}

In this section, we develop the power-capacity relationship of both SHIP and the repetition scheme for forward-link communications. Both the 2-D and the linear cell models are studied. We assume that the MNs are uniformally distributed over all cells with density $\eta$.

\section{A. Power Control Solution}

In a forward-link communication, the amount of interference $\sum_{m \in \mathcal{A}_{k}} \mathcal{I}_{i m}$ experienced by a $\mathrm{MN}_{i}$ situated in $\mathrm{AN}_{k}$ can be evaluated as

$$
\sum_{m \in \mathcal{A}_{k}} \mathcal{I}_{i m}=\sum_{m \in \mathcal{A}_{k}} \mathcal{H}_{i m} \sum_{j \in \mathrm{AN}_{m}} P_{j m} .
$$

Since the MNs are uniformally distributed and the MN density is the same for all cells, the amount $\sum_{j \in \mathrm{AN}_{m}} P_{j m}$ is the same for all $\mathrm{AN}_{m}, m \in \mathcal{A}_{k}$. Let $\mathcal{P}_{\mathcal{T}}$ be that amount. Note that $\mathcal{P}_{\mathcal{T}}$ designates the total amount of power consumed by any $\mathrm{AN}_{m}, m \in \mathcal{A}_{k}$. Thus, (4) can be rewritten as

$$
\sum_{m \in \mathcal{A}_{k}} \mathcal{I}_{i m}=\mathcal{P}_{\mathcal{T}} \sum_{m \in \mathcal{A}_{k}} \mathcal{H}_{i m}
$$

Therefore, the power control problem stated by (3) can be redefined as: For every $\mathrm{MN}_{i} \in \mathrm{AN}_{k}$, find the power $P_{i k}$ minimizing $\mathcal{P}_{\mathcal{T}}=\sum_{j \in \mathrm{AN}_{k}} P_{j k}$ such that

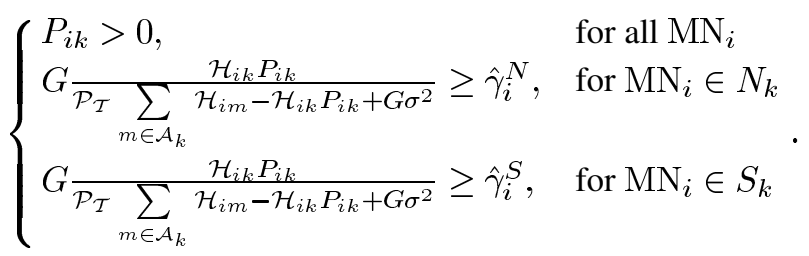

Theorem 1: Let $\hat{\omega}_{i}^{N}=\hat{\gamma}_{i}^{N} /\left(G+\hat{\gamma}_{i}^{N}\right)$, and $\hat{\omega}_{i}^{S}=$ $\hat{\gamma}_{i}^{S} /\left(G+\hat{\gamma}_{i}^{S}\right)$ for all $\mathrm{MN}_{i} \in \mathrm{AN}_{k}$. The power control optimization problem stated by (5) has a unique solution defined by

$$
P_{i k}= \begin{cases}\frac{\hat{\omega}_{i}^{N}}{\mathcal{H}_{i k}}\left(\xi_{i} \mathcal{P}_{\mathcal{T}}+G \sigma^{2}\right), & \text { for } \mathrm{MN}_{i} \in N_{k} \\ \frac{\hat{\omega}_{i}^{S}}{\mathcal{H}_{i k}}\left(\xi_{i} \mathcal{P}_{\mathcal{T}}+G \sigma^{2}\right), & \text { for } \mathrm{MN}_{i} \in S_{k}\end{cases}
$$

where

$$
\mathcal{P}_{\mathcal{T}}=\frac{G \sigma^{2}\left\{\sum_{i \in N_{k}} \frac{\hat{\omega}_{i}^{N}}{\mathcal{H}_{i k}}+\sum_{i \in S_{k}} \frac{\hat{\omega}_{i}^{S}}{\mathcal{H}_{i k}}\right\}}{1-\sum_{i \in N_{k}} \hat{\omega}_{i}^{N} \frac{\xi_{i}}{\mathcal{H}_{i k}}-\sum_{i \in S_{k}} \hat{\omega}_{i}^{S} \frac{\xi_{i}}{\mathcal{H}_{i k}}}
$$

and

$$
\xi_{i}=\sum_{m \in \mathcal{A}_{k}} \mathcal{H}_{i m}
$$




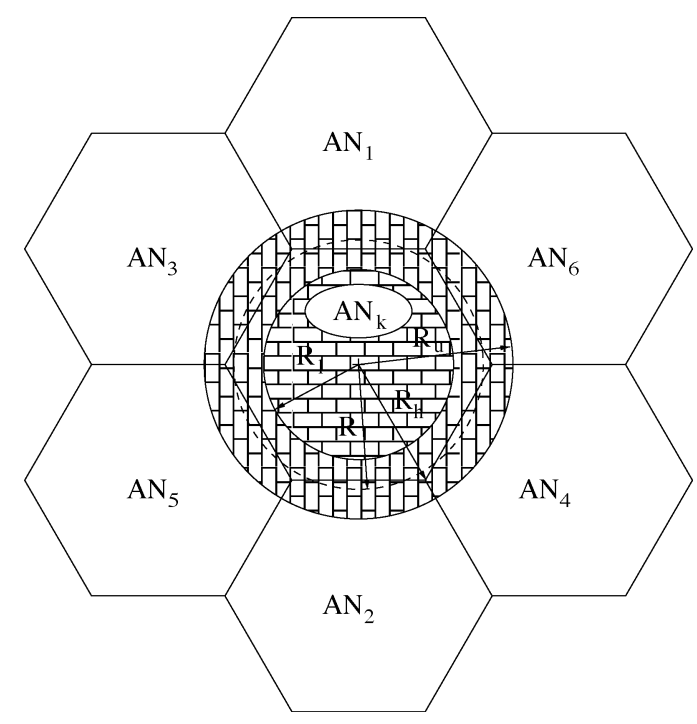

Fig. 8. Interfering cells: 2-D model.

under the condition

$$
1-\sum_{i \in N_{k}} \hat{\omega}_{i}^{N} \frac{\xi_{i}}{\mathcal{H}_{i k}}-\sum_{i \in S_{k}} \hat{\omega}_{i}^{S} \frac{\xi_{i}}{\mathcal{H}_{i k}}>0
$$

Remark 1: The proof of Theorem 1 is given in Appendix I.

\section{B. Power-Capacity Relationship: 2-D Model}

In this section, we consider the 2-D cell model which consists of one studied cell $k$ situated in between neighbor cells as shown in Fig. 8. Different geometrical cell shapes have been studied [1], [3], [4], [14]-[17], where the hexagonal/circular cell structure is mostly used. Depending on the studied objective and performance, people have characterized the soft handoff region differently. We define the soft handoff zone of $\mathrm{AN}_{k}$ to be the region lower-bounded and upper-bounded, respectively, by the circles of radius $R_{l}$ and $R_{u}$ (see Fig. 8). $R_{h}$ denotes the radius of the hexagonal cells which is defined as the furthest point from the center. $R=(3 \sqrt{3} / \pi)^{(1 / 2)} R_{h}$ is defined to be the radius of the disk equicentred and having an identical area to the hexagonal cell. Let $r=a / R$ be a characterization of the portion of the soft handoff zone; where $a=R_{u}-R=R-R_{l}$.

We assume that $\mathrm{AN}_{k}$ interferes only with its six direct neighbor cells $\mathrm{AN}_{m}, m \in\{1,2,3,4,5,6\}$. We also assume that the targets $\hat{\gamma}_{i}^{N}$ and $\hat{\gamma}_{i}^{S}$ are the same for all $\mathrm{MN}_{i}$ communicating from $N_{k}$ and $S_{k}$, respectively, and so are $\hat{\omega}_{i}^{N}$ and $\hat{\omega}_{i}^{S}$.



Fig. 9. Interfering cells: linear model.

Therefore, we will refer to $\hat{\omega}_{i}^{N}$ as $\hat{\omega}^{N}$ and $\hat{\omega}_{i}^{S}$ as $\hat{\omega}^{S}$. From (7), the $\mathrm{MN}$ density can be rewritten as (8), shown at the bottom of the page, where for both SHIP and the repetition soft handoff scheme

$$
\begin{aligned}
& \chi_{\text {noise }}^{N}(r, \alpha)=2(1-r)^{\alpha+2} \\
& \chi_{\text {power }}^{N}(r, \alpha)=(1-r)^{2}+6 \int_{s=0}^{s=1-r} \int_{t=0}^{t=2} \mathcal{F}_{\alpha}(s, t) d s d t \\
& \chi_{\text {noise }}^{S}(r, \alpha)=2(1+r)^{\alpha+2}-2(1-r)^{\alpha+2} \\
& \chi_{\text {power }}^{S}(r, \alpha)=4 r+6 \int_{s=1-r}^{s=1+r} \int_{t=0}^{t=2} \mathcal{F}_{\alpha}(s, t) d s d t
\end{aligned}
$$

with

$$
\mathcal{F}_{\alpha}(s, t)=\frac{s^{\alpha+1}}{\left(s^{2}-2\left(\frac{\pi}{\sqrt{3}}\right)^{\frac{1}{2}} s \sin (\pi t)+\frac{\pi}{\sqrt{3}}\right)^{\frac{\alpha}{2}}} .
$$

Remark 2: The derivation of (8) is given in Appendix II-A.

\section{Power-Capacity Relationship: Linear Model}

We also consider the linear cell model in this work. In the linear model, we assume that the studied cell $k$ interferes only with its two closest neighbor cells (the right cell and the left cell), as shown in Fig. 9. Let $\mathrm{AN}_{1}$ and $\mathrm{AN}_{2}$ denote these two interfering cells; i.e., $\mathcal{A}_{k}=\{1, k, 2\}$. When a $\mathrm{MN}_{i}$ enters $N_{k}$, it connects only to $\mathrm{AN}_{k}$ whereas, if it enters $S_{k}$ on the side of $\mathrm{AN}_{1}$, the $\mathrm{MN}_{i}$ is allowed to simultaneously communicate with $\mathrm{AN}_{k}$ and $\mathrm{AN}_{1}$.

Let $r=a / L$ be the ratio of the length of the soft handoff zone to that of the whole cell, where $a$ is half the length of the soft handoff zone. The linear density $\eta$ of the MNs as a function of the total power $\mathcal{P}_{\mathcal{T}}$ and the ratio $r$ can be derived from (7) and written as (9), shown at the bottom of the page, where for both

$$
\eta\left(\mathcal{P}_{\mathcal{T}}, r\right)=\frac{\mathcal{P}_{\mathcal{T}}}{\frac{G \sigma^{2} \pi R^{\alpha+2}}{\alpha+2}\left\{\hat{\omega}^{N} \chi_{\text {noise }}^{N}(r, \alpha)+\hat{\omega}^{S} \chi_{\text {noise }}^{S}(r, \alpha)\right\}+\mathcal{P}_{\mathcal{T}} \pi R^{2}\left\{\hat{\omega}^{N} \chi_{\text {power }}^{N}(r, \alpha)+\hat{\omega}^{S} \chi_{\text {power }}^{S}(r, \alpha)\right\}}
$$

$$
\eta\left(\mathcal{P}_{\mathcal{T}}, r\right)=\frac{\mathcal{P}_{\mathcal{T}}}{\frac{G \sigma^{2} L^{\alpha+1}}{\alpha+1}\left\{\hat{\omega}^{N} \beta_{\text {noise }}^{N}(r, \alpha)+\hat{\omega}^{S} \beta_{\text {noise }}^{S}(r, \alpha)\right\}+\mathcal{P}_{\mathcal{T}} L\left\{\hat{\omega}^{N} \beta_{\text {power }}^{N}(r, \alpha)+\hat{\omega}^{S} \beta_{\text {power }}^{S}(r, \alpha)\right\}}
$$


TABLE I

SUMMARY OF PARAMETERS

\begin{tabular}{l|c}
\hline \hline Parameters & Values \\
\hline \hline Cell length, $\mathrm{L}, \mathrm{R}$ & $400 \mathrm{~m}$ \\
\hline Packet length, $\mathcal{L}$ & 128 symbols \\
\hline Symbol length, $\mathcal{S}$ & 8 bits \\
\hline Variance of noise, $\sigma^{2}$ & $10^{-13}$ \\
\hline Processing gain, $\mathrm{G}$ & 200 \\
\hline Path loss factor, $\alpha$ & 2 \\
\hline Total power, $\mathcal{P}_{\mathcal{T}}$ & $100 \mathrm{~mW}$ \\
\hline \hline
\end{tabular}

schemes

$$
\begin{aligned}
& \beta_{\text {noise }}^{N}(r, \alpha)=2(1-r)^{\alpha+1} \\
& \beta_{\text {power }}^{N}(r, \alpha)=2(1-r)+2 \int_{0}^{1-r} \frac{(2-l)^{-\alpha}+(2+l)^{-\alpha}}{l^{-\alpha}} d l \\
& \beta_{\text {noise }}^{S}(r, \alpha)=2(1+r)^{\alpha+1}-2(1-r)^{\alpha+1} \\
& \beta_{\text {power }}^{S}(r, \alpha)=4 r+2 \int_{1-r}^{1+r} \frac{(2-l)^{-\alpha}+(2+l)^{-\alpha}}{l^{-\alpha}} d l
\end{aligned}
$$

Remark 3: The derivation of (9) is given in Appendix II-B.

Notice that if the noisy term, present in the denominator of the power-capacity relations given by (8) and (9), is negligible with respect to the power term, then $\eta\left(\mathcal{P}_{\mathcal{T}}, r\right)$ can be simplified to $\eta(r)$; meaning that the capacity $\eta(r)$ does not depend on the total power $\mathcal{P}_{\mathcal{T}}$. This observation will be discussed in Section V. Also notice that the more significant the noise of the medium, the worse the effect of $\beta_{\text {noise }}$ or $\chi_{\text {noise }}$ on the capacity. In other words, if the noise is negligible, then a variation of $\beta_{\text {noise }}$ or $\chi_{\text {noise }}$ does not affect the capacity.

\section{EMPIRICAL EVALUATION OF SHIP}

In this section, we empirically evaluate the performance of the network during a forward-link traffic for the 2-D and the linear cell models. SHIP and the repetition soft handoff scheme are analyzed and compared with the hard-handoff scheme. As mentioned in Section III-B, the path loss model used in this work is $\mathcal{H}_{i k}=l^{-\alpha}$, where $\alpha \in\{1,2,3, \ldots\}$. We use $\alpha=2$. Table I presents the parameters used in the evaluation.

To compare the schemes, we proceed as follows. The network imposes the same target PER $\widehat{\Upsilon}$ for all the three schemes. Given $\widehat{\Upsilon}$, there is a tradeoff between the total consumed power $\mathcal{P}_{\mathcal{T}}$ and the network capacity $\eta$ as depicted in (8) and (9). We choose the total transmit power $\mathcal{P}_{\mathcal{T}}$ to be $120 \mathrm{~dB}$ as compared with the noise power. The following two metrics are used in this evaluation.

- PER, $\Upsilon$ : It is the probability that a received packet is erroneous. A packet is considered erroneous when at least 1 bit is incorrect after combining.

- Density, $\boldsymbol{\eta}$ : It is the MN density over the cells. The density $\eta$ is the number of MNs per unit of surface (respectively, unit of length) in the 2-D (respectively, linear) cell model. The density $\eta$ is considered constant for all the cells.

Figs. 10 and 11 show the effect of the soft handoff zone ratio $r$ on the capacity of the system for both the 2-D and the linear cell models. Recall that $r$ is the ratio characterizing the portion of the soft handoff zone of that of the whole cell. Figs. 12 and 13 plot the density gain/loss of the network as

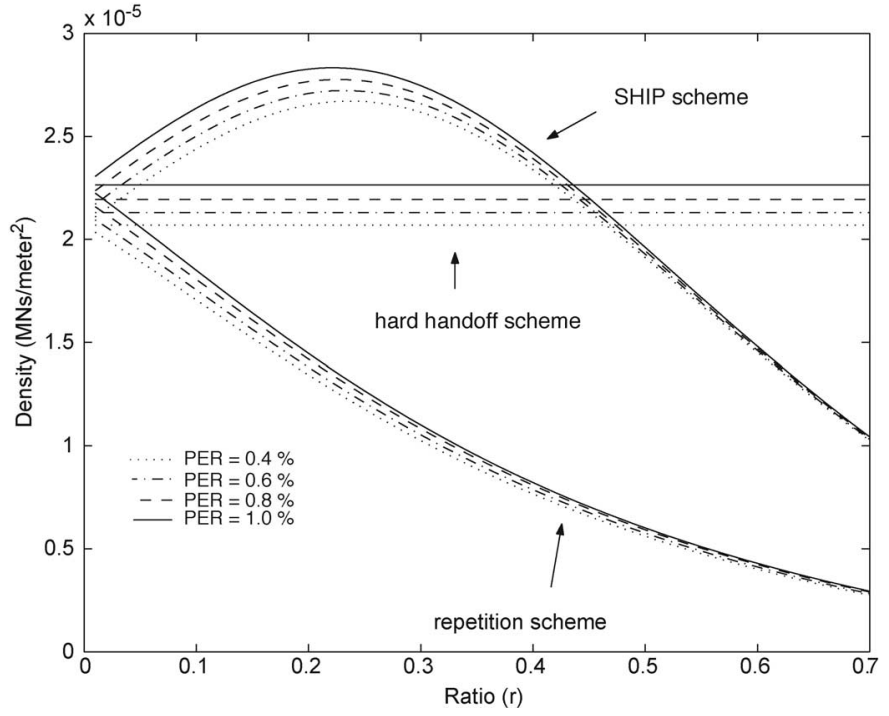

Fig. 10. Density $\eta$ as a function of the ratio $r$ : 2-D model.

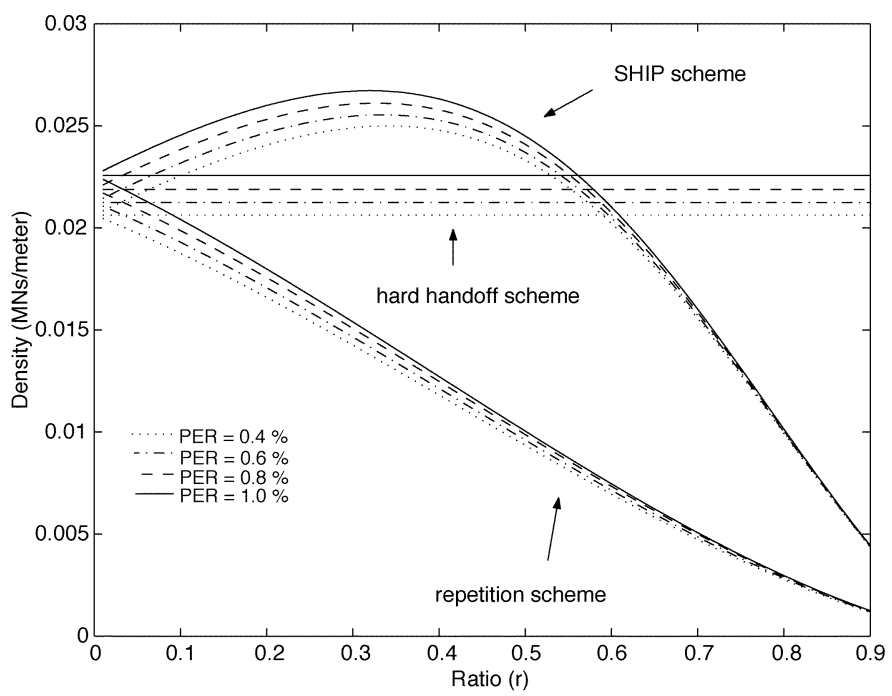

Fig. 11. Density $\eta$ as a function of the ratio $r$ : linear model.



Fig. 12. Density gain/loss as a function of the ratio $r$ : 2-D model.

a function of the ratio $r$. Results are given for a set of PERs, $\widehat{\Upsilon} \in\{1 \%, 0.8 \%, 0.6 \%, 0.4 \%\}$. As mentioned in Section IV-C, 


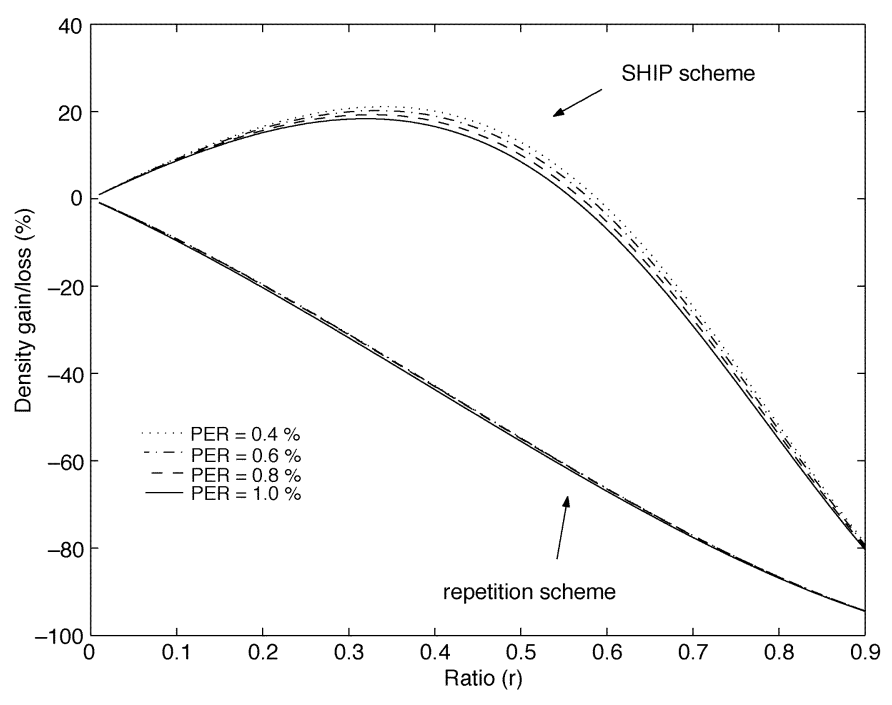

Fig. 13. Density gain/loss as a function of the ratio $r$ : linear model.

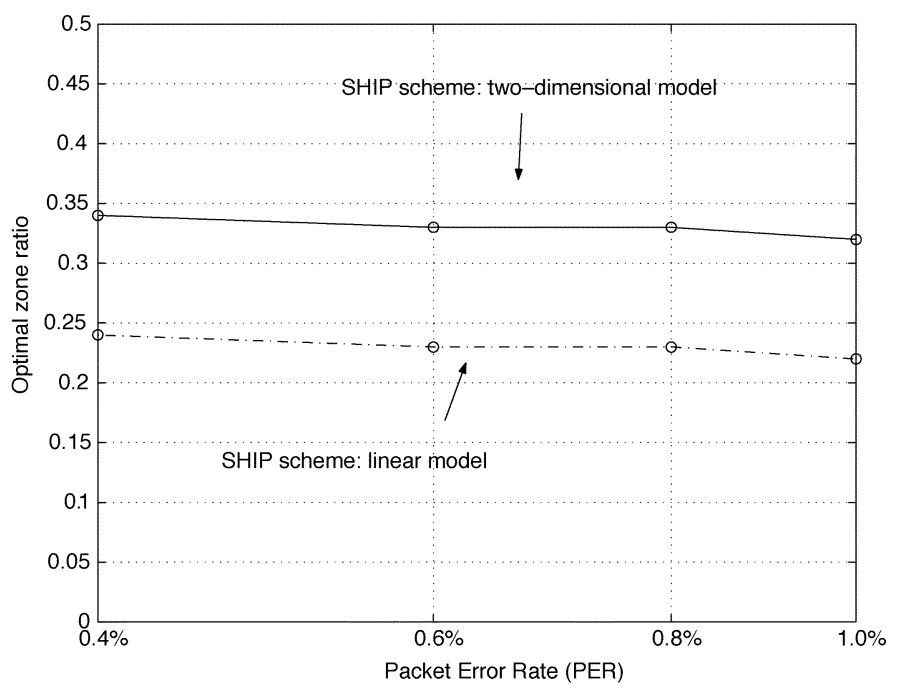

Fig. 14. Optimal handoff zone ratio $r_{\mathrm{opt}}$ as a function of the PER, $\Upsilon$.

since the noisy term present in (8) and (9) is negligible with respect to the power term, then a variation of $\mathcal{P}_{\mathcal{T}}$ does not affect the capacity gain/loss. Thus, the results presented in Figs. 10-13 are $\mathcal{P}_{\mathcal{T}}$-independent. For the hard handoff, $\eta$ does not depend on $r$. However, the capacity is a strong function of $r$ for both soft handoff schemes. Observe that the capacity is less than hard handoff for the repetition scheme for all values of $r$. SHIP, on the other hand, has higher capacity than the other two schemes for a large range of $r$. This result is shown in terms of percentage gain/loss with respect to the hard handoff scheme in Figs. 12 and 13 for the 2-D and the linear models.

Fig. 14 shows the optimal soft handoff zone ratio $r_{\text {opt }}$ for different target PERs. It is worth noting that the lower the PER, the higher the ratio, $r_{\mathrm{opt}}$. For typical values of $\widehat{\Upsilon}$, the optimal ratio $r_{\text {opt }}$ is between 0.30 and 0.35 for the 2-D model and between 0.20 and 0.25 for the linear model.

Fig. 15 depicts the density gain/loss as a function of the PER for both cell models. SHIP gains up to $\approx 30 \%$ in the capacity for the 2-D model and up to $\approx 20 \%$ for the linear cell model. This

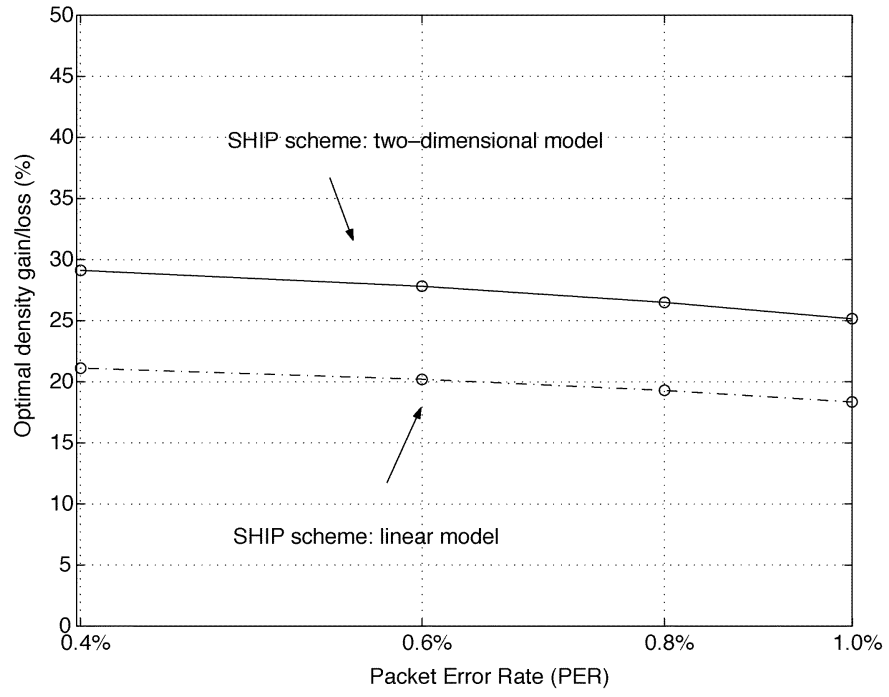

Fig. 15. Density gain/loss of SHIP at the optimal ratio $r_{\mathrm{opt}}$.

gain is reached for an optimal zone ratio $r_{\text {opt }}$ of $\approx 0.34$ and $\approx 0.22$, respectively, for the $2-\mathrm{D}$ and the linear models.

\section{SimUlation OF SHIP}

The empirical evaluation of the capacity gain of SHIP conducted in Section V was performed under the assumption that the distribution of the MNs is uniform. To remove the speculation of whether this assumption is reasonable, we also evaluate the performance of the soft handoff schemes through simulations. During the simulation runs, we measure the total amount of power $\mathcal{P}_{\mathcal{T}}$ consumed by the studied cell and use it as the performance metric of the evaluation. We evaluate the effectiveness of SHIP by comparing the total power gain/loss of both soft handoff schemes with respect to the hard handoff scheme. The simulation parameters are set to those given in Table I unless stated otherwise.

\section{A. Simulation Method}

To simulate the 2-D model, we consider a cell $k$ surrounded from all directions by a large number of cells so that the boundary effect is neglected. We consider an area of $5 \times 5 \mathrm{~km}$ square, where cell $k$ is located in the center. The radius $R$ of each cell is set to $400 \mathrm{~m}$. For the linear model, we consider 100 adjacent cells each of length $2 L=800 \mathrm{~m}$, where the studied cell $k$ is placed in the middle. In both models, the studied cell $k$ interferes only with its immediate neighbor cells. In the 2-D model, we assume that MNs do not move - even if they do, we believe that their mobility does not affect the total consumed power. Unlike the 2-D model, MNs in the linear model move at random speeds.

Initially, there are $\mathcal{N}_{\text {avg }}$ MNs uniformally distributed at random locations in the system. ${ }^{1}$ Every second, a MN 2 enters the system at a uniformally selected location with probability $p_{\text {arrival }}=0.8$. In the linear model, upon its entrance to the

${ }^{1}$ The term system refers to the city or town in the case of the 2-D model, and to the highway in the case of the linear model.

${ }^{2}$ In this section, the term MN refers to a communication; i.e., an entering $\mathrm{MN}$ could be thought of as a new call if MNs are cellular phones. 


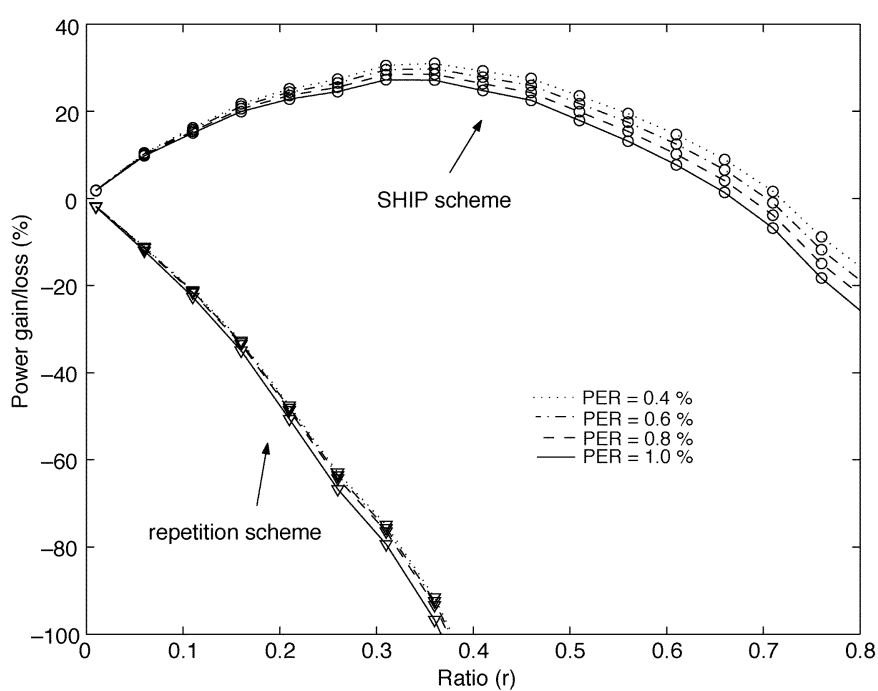

Fig. 16. Power gain/loss as a function of $r: 2$-D model, $\mathcal{N}_{\text {avg }}=15$.

system (either initially or later on), the $\mathrm{MN}$ is also associated with a random direction and a random speed selected uniformally from an interval $[60 \mathrm{~km} / \mathrm{h}, 120 \mathrm{~km} / \mathrm{h}]$. Every second, each $\mathrm{MN}$ is removed from the system with probability $p_{\text {departure }}$ which leads to an expected lifetime of $1 / p_{\text {departure }}$ seconds. ${ }^{3}$ MNs in the linear system move at their selected speeds until they either leave the cells or are removed from the system.

\section{B. Simulation Results}

We conducted simulations for different values of $\mathcal{N}_{\text {avg }} \in\{10,15,20,25,30,35,40\}$. To maintain system stability, the departure probability is chosen such that $\mathcal{N}_{\text {avg }}$ equals $p_{\text {arrival }} / p_{\text {departure }}$. For each scenario, 50 simulations are performed each of which runs for 12 hours. Power measurements are taken at random instants according to a Poisson process with rate 0.75 . The results are averaged over all simulations.

In Figs. 16 and 17, we show the total power gain/loss of both soft handoff schemes relative to the hard handoff scheme when the average number $\mathcal{N}_{\text {avg }}$ of MNs is equal to 15 . Results are given for PER equals to $1 \%, 0.8 \%, 0.6 \%$, and $0.4 \%$. Note that SHIP saves up to $30 \%$ and $20 \%$ of the total power for the 2-D and linear models, respectively, whereas the repetition scheme actually looses power. Fig. 18 shows that the gain in power is also maintained when the average number of MNs is varied. The figure illustrates the gain in power when the ratio $r$ is set to the optimal ratio $r_{\text {opt }}$ that corresponds to $\mathcal{N}_{\text {avg }}=15$. The PER is set to $0.8 \%$.

In conclusion, through these simulation studies, we confirm that SHIP results in substantial improvements of the performance of the network. These improvements can be expressed either in power savings or in capacity increase.

\section{IMPLEMENTATION OF SHIP}

SHIP is implemented in the network stack of the Linux Kernel v2.4.18-3 from Red Hat. The first phase involves

${ }^{3}$ This results in geometrically distributed lifetimes of MNs with parameter $p_{\text {departure }}$.

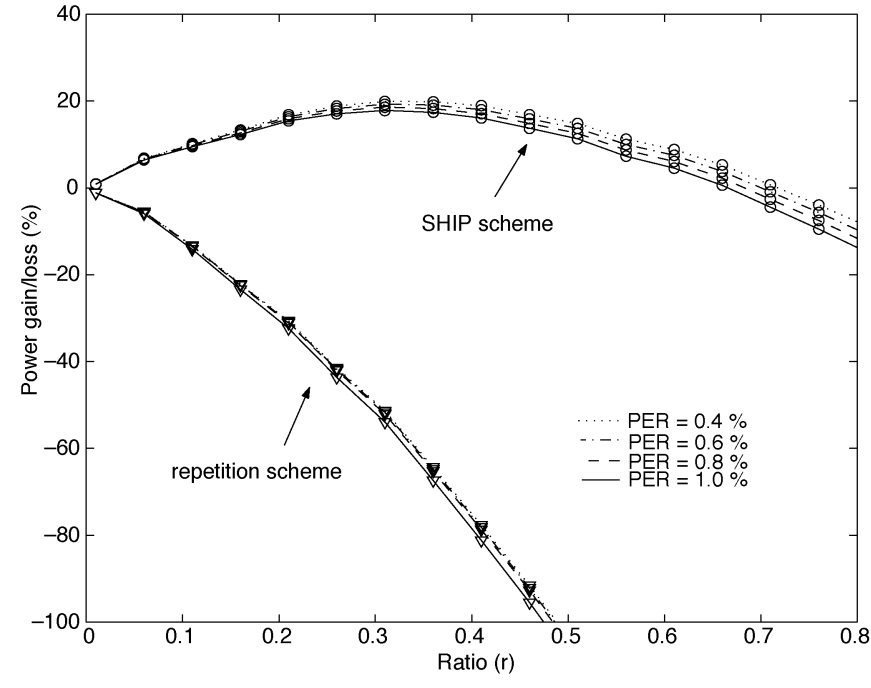

Fig. 17. Power gain/loss as a function of $r$ : linear model, $\mathcal{N}_{\text {avg }}=15$.

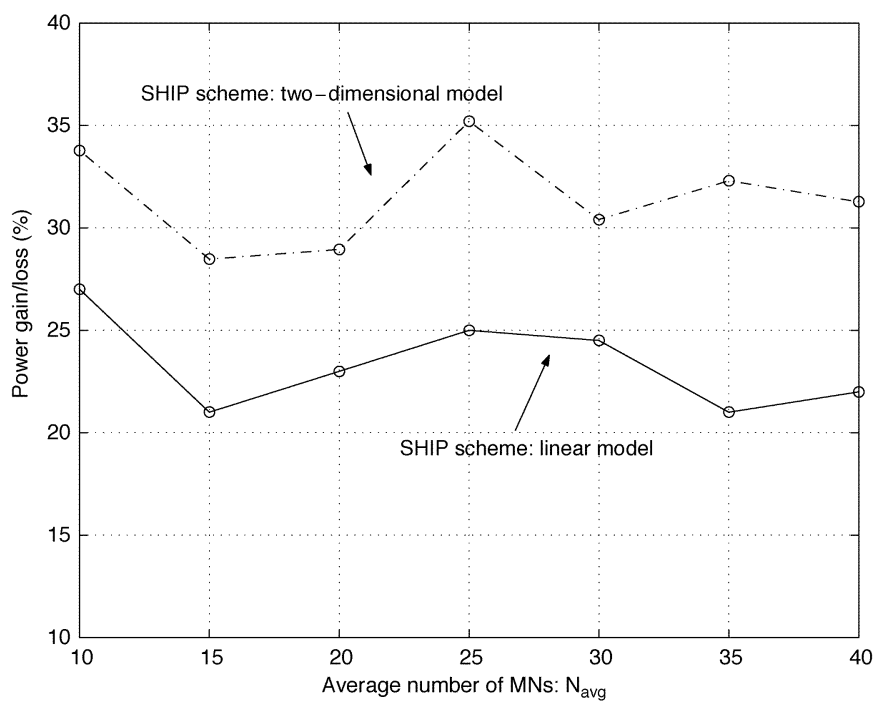

Fig. 18. Power gain/loss as a function of the average number $\mathcal{N}_{\text {avg }}$ of MNs.

the implementation of the SHIP-DU at the SHIP-FA, as described in Section II-B1. The SHIP-DU module is inserted between the network-layer and the link-layer. SHIP is implemented as a module that can be inserted into or removed from the kernel without having to restart. The module pointer is inserted at the beginning of the link-layer (/usr/src/linux-2.4.18-3/net/core/dev.c) to where packets coming from the network-layer are passed. Packets destined to MNs under soft handoff are generated into two different descriptions and put back into the queue for transmission. Packets destined to MNs not under soft handoff mode bypass the SHIP-DU module. The second phase involves the implementation of the SHIP Combiner Unit at the MN as described in Section II-B2.

Fig. 19 illustrates the testbed used in this work. Two CISCO Aironet 1200 access points are used as ANs. A Linux machine (wallaby.ece.wisc.edu), connected to both access points via Ethernet, is used as the forwarding agent. Two other machines (ferrari.ece.wisc.edu) and (springbuck.ece.wisc.edu) both equipped 


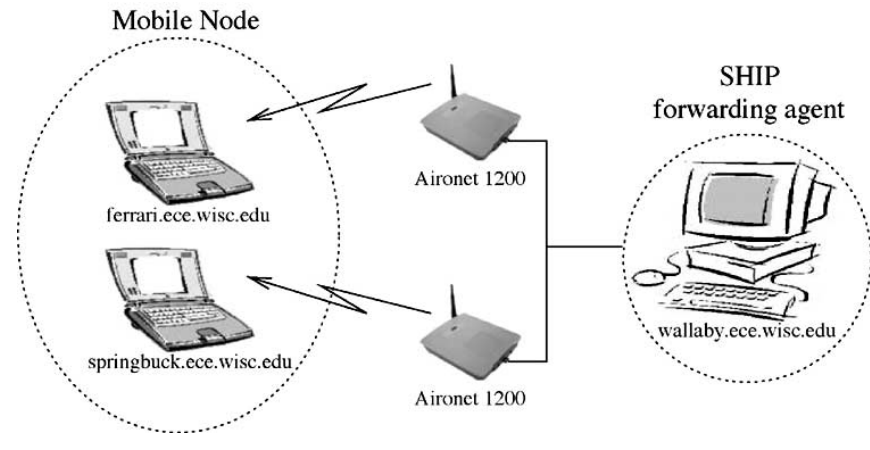

Fig. 19. Experimental testbed.

with Orinoco IEEE $802.11 \mathrm{~b}$ PC cards are used to emulate a single MN with two different wireless cards. We used two machines instead of one because the Kernel implementation does not allow inserting two wireless cards on the same machine. Since a single MN will have one IP address in SHIP, we assigned the same IP address to both machines.

Two descriptions are generated by the inserted module for each packet destined to the emulated MN. The two descriptions delivered to the two machines (springbuck.ece.wisc.edu and ferrari.ece.wisc.edu) via different access points. By setting the access points with different power levels and varying the distance between the access points and the MN, one can measure the dropping rate seen with and without the implementation of SHIP. However, in the system, the physical-layer drops most of the erroneous packets. Since the primary gain in SHIP comes from combining erroneous received descriptions, the benefits of SHIP can not be demonstrated. A SHIP-aware physical-layer can implement significantly less error correction scheme and, thus, increase the data rate. Moreover, SHIP further decreases the retransmission rate since more packets will be corrected by the Reed-Solomon codes at the network-layer, thus preventing higher layers such as TCP from retransmission. Furthermore, the IEEE $802.11 \mathrm{~b}$ is not a CDMA-based medium access scheme. Due to these reasons, it is not possible to validate the capacity gain or the power savings derived in Sections $\mathrm{V}$ and VI by using our experimental testbed. The experimental testbed, however, demonstrates the ease of implementation of the proposed scheme.

\section{LATENCY OF SHIP}

In practice, the timeout value of the SHIP timer must be large enough to assure that both descriptions arrive at the MN before the timer expires. The worse-case time difference between the arrival times of the two descriptions occurs when one description encounters an empty link-layer queue, while the other encounters an almost full queue. This means that the timeout should be larger than the time required to deliver a description through an almost full queue. In practice, we expect this timeout value to be much larger than the largest value of all factors influencing the worst-case latency. ${ }^{4}$ In particular, the time required to generate the two descriptions and the time required to combine

\footnotetext{
${ }^{4}$ The latency is defined as the difference between the time a packet is delivered to the SHIP-DU at the SHIP-FA and the time the packet is passed to the IP processing and routing unit at the MN.
}

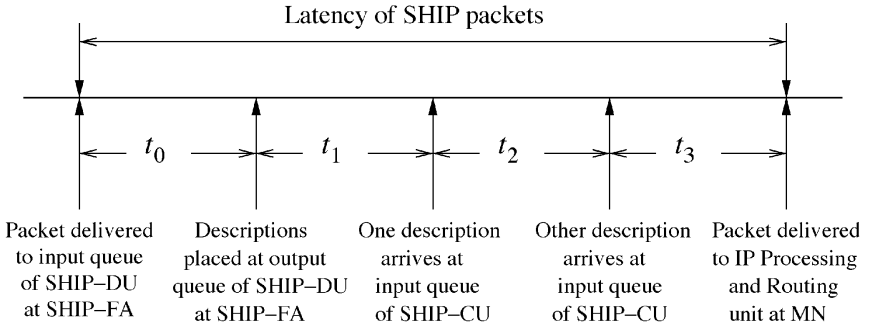

(a)

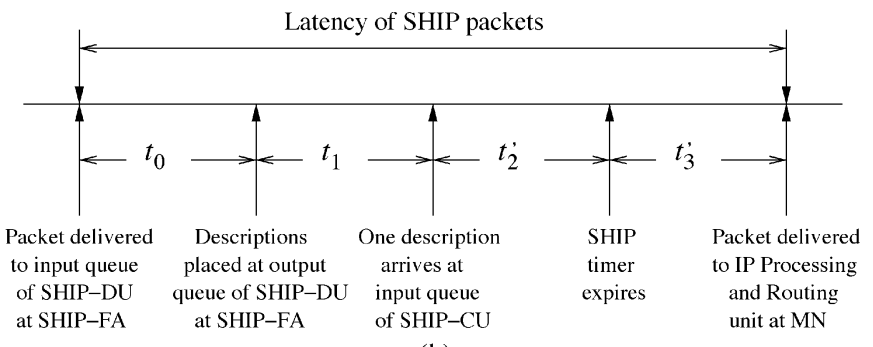

(b)

Fig. 20. Latency of SHIP packets: sequence of events. (a) Normal delivery of SHIP packets. (b) Erroneous delivery of SHIP packets.

them are not likely to significantly determine the worst-case latency of delivering SHIP packets. Instead, the worst-case latency is mainly determined by the expected heaviest load on the ANs. Note that this is also the worst-case latency on the hard handoff scheme. In other words, the worst-case latency of SHIP is approximately equal to the worst-case latency of the hard handoff scheme.

In this section, we characterize the latency experienced by packets in SHIP. Fig. 20 shows the sequence of events that occur in normal and erroneous conditions in delivering packets in SHIP. In normal circumstances [Fig. 20(a)], i.e., when both descriptions arrive at the SHIP Combiner Unit before the SHIP timer expires, the latency is $t_{0}+t_{1}+t_{2}+t_{3}$ where $t_{0}$ is the time required to generate the Reed-Solomon description; $t_{1}$ is the queueing delay at the ANs and the packet transmission time (from SHIP-FA to ANs and from ANs to MN); $t_{2}$ is the time difference between the arrival of the two descriptions due to differences in the queueing delays at the ANs; and $t_{3}$ is the time required to generate a packet from the two descriptions using the Reed-Solomon decoder. In this latency, we expect $t_{1}+t_{2}$ to be much larger than $t_{0}+t_{3}$. Note that the worst-case latency of hard handoff packets is also determined by the amount $t_{1}+t_{2}$. In some instances, one of the description may not arrive due to buffer overflow at link-layer of the ANs or improper reception at the MN. In this case, timeout will occur and the SHIP-CU will assume that the second description is all $0 \mathrm{~s}$, decode both descriptions, and forward the decoded packet to the IP processing and routing unit. The latency in this case is $t_{0}+t_{1}+t_{2}^{\prime}+t_{3}^{\prime}$ [see Fig. 20(b)]

\section{CONCLUSION}

In this paper, we propose, analyze, simulate, and implement a soft handoff approach for wireless IP-based networks. The proposed scheme eliminates the need for synchronizing the ANs simultaneously transmitting to the MNs during forward-link soft handoff communications. The goal is achieved by percolating 
the received descriptions of each packet up to the network-layer instead of traditionally being at the physical-layer. In addition, the proposed soft handoff scheme increases the capacity, and/or saves the consumed power of the network in the forward-link traffic by exploiting efficiently the repetition of the descriptions. We analytically derive the capacity of the network for hard handoff, SHIP, and the repetition soft handoff scheme. We show that the proposed technique achieves substantially better performance improvements than the repetition scheme. Empirically, we show that the forward-link network capacity is increased by about $30 \%$ and $20 \%$ as compared with the hard handoff, respectively, for the 2-D and the linear models. Further, through simulation studies, we prove that when SHIP is used, the ANs could save up to $30 \%$ of the total consumed power. Through an experimental testbed, we demonstrate the ease of implementation of SHIP.

\section{APPENDIX I}

\section{PROOF OF THEOREM 1}

We need to prove that the power vector $P_{i k}$ defined by (6) is (i) a solution to the problem and (ii) unique. Let $(a),(b)$, and $(c)$ denote, respectively, the first, the second, and the third inequalities given in (5).

Existence: To prove $(i)$, it suffices to show that the power vector $P_{i k}$ satisfies the three conditions $(5)(a)-(5)(c)$ and minimizes the total power. Condition $(5)(a)$ is automatically satisfied by the condition imposed on the denominator. By replacing $P_{i k}$ and $\mathcal{P}_{\mathcal{T}}$ by their expressions given, respectively, in (6) and (7), we easily find that $\gamma_{i}=\hat{\gamma}_{i}^{N}$ and $\gamma_{i}=\hat{\gamma}_{i}^{S}$ for $\mathrm{MN}_{i} \in N_{k}$ and $\mathrm{MN}_{i} \in S_{k}$. This implies that conditions (5)(b) and $(5)(c)$ are satisfied. Now, we need to show that $P_{i k}$ minimizes the total power. Suppose there exists a power vector $P_{i k}^{*}$, $\mathrm{MN}_{i} \in \mathrm{AN}_{k}$, satisfying the three conditions (5)(a)-(5)(c) such that $\sum_{j \in \mathrm{AN}_{k}} P_{j k}^{*}<\sum_{j \in \mathrm{AN}_{k}} P_{i k}$. As a result, $\exists \mathrm{MN}_{j} \in \mathrm{AN}_{k}$ such that $P_{j k}^{*}<P_{j k}$. If $\mathrm{MN}_{j} \in N_{k}$ (with respect to $\in S_{k}$ ), the condition $(5)(b)$ (with respect to $(5)(c)$ ) will be violated since the resulting $\gamma_{i}^{*}$ of $\mathrm{MN}_{i}$ will fall below the target $\hat{\gamma}_{i}^{N}$ (with respect to $\hat{\gamma}_{i}^{S}$ ). Thus, the assumption is absurd.

Uniqueness: To prove (ii), suppose there exists another solution $P_{i k}^{-}, \mathrm{MN}_{i} \in \mathrm{AN}_{k}$, to the problem different from $P_{i k}$ given by (6). That is, $\exists \mathrm{MN}_{j} \in \mathrm{AN}_{k}$ such that $P_{j k}^{-} \neq P_{j k}$. If $P_{i k}^{-}<$ $P_{i k}$, then either the condition $(5)(b)$ or the condition $(5)(c)$ will be violated. If $P_{i k}^{-}>P_{i k}$, then $\sum_{j \in \mathrm{AN}_{k}} P_{j k}^{-}>\sum_{j \in \mathrm{AN}_{k}} P_{i k}$ which means that the solution $P_{i k}^{-}$does not minimize the total power and, thus, it is not even a solution. Consequently, the solution is unique.

\section{APPENDIX II}

\section{DERIVATION OF THE POWER-CAPACITY RELATIONSHIP}

Let $\Omega_{1}, \quad \Omega_{2}, \quad \Omega_{3}$ and $\Omega_{4}$ be $\sum_{i \in N_{k}}\left(\hat{\omega}_{i}^{N} / \mathcal{H}_{i k}\right)$, $\sum_{i \in S_{k}}\left(\hat{\omega}_{i}^{S} / \mathcal{H}_{i k}\right), \quad \sum_{i \in N_{k}} \hat{\omega}_{i}^{N}\left(\xi_{i} / \mathcal{H}_{i k}\right)$ and $\sum_{i \in S_{k}} \hat{\omega}_{i}^{S}$ $\left(\xi_{i} / \mathcal{H}_{i k}\right)$, respectively. Thus, (7) can be written as

$$
\mathcal{P}_{\mathcal{T}}=\frac{G \sigma^{2}\left(\Omega_{1}+\Omega_{2}\right)}{1-\Omega_{3}-\Omega_{4}} .
$$

Recall that $\hat{\omega}_{i}^{N} \equiv \hat{\omega}^{N}$ and $\hat{\omega}_{i}^{S} \equiv \hat{\omega}^{S}$ are assumed to be the same for all $\mathrm{MN}_{i} \in N_{k}$ and $\mathrm{MN}_{i} \in S_{k}$. The path loss $\mathcal{H}_{i m}$ between
$\mathrm{MN}_{i}$ and an access node $\mathrm{AN}_{m}$ is defined to be $d^{-\alpha}$, where $d$ is the distance between $\mathrm{MN}_{i}$ and $\mathrm{AN}_{m}$.

\section{A. Two-Dimensional Model}

Let $d S$ be a surface element of the cell $k$, and $d N$ be the number of MNs in $d S$; i.e., $\eta=d N / d S$. As a first step, we can write $\Omega_{1}=\hat{\omega}^{N} \iint_{N_{k}} \rho^{\alpha} d N=\hat{\omega}^{N} \eta \iint_{N_{k}} \rho^{\alpha} d S$ and $\Omega_{2}=$ $\hat{\omega}^{S} \iint_{S_{k}} \rho^{\alpha} d N=\hat{\omega}^{S} \eta \iint_{S_{k}} \rho^{\alpha} d S$; where $\rho$ is the distance between $d S$ and $\mathrm{AN}_{k}$.

For the 2-D model (see Fig. 8), the element $d S$ can be evaluated as $\rho d \rho d \theta$, where $0 \leq \theta \leq 2 \pi$ and $0 \leq \rho \leq R_{l}$ (with respect to $R_{l} \leq \rho \leq R_{u}$ ) for the zone $N_{k}$ (with respect to $S_{k}$ ). Now, consider the following change of variable $\tau=\rho / R$. Given that $r=a / R=\left(R_{u}-R\right) / R=\left(R-R_{l}\right) / R$, by a simple calculation, we can write

$$
\Omega_{1}=\frac{\eta \hat{\omega}^{N} \pi R^{\alpha+2}}{(\alpha+2)}\left\{2(1-r)^{\alpha+2}\right\}
$$

and

$$
\Omega_{2}=\frac{\eta \hat{\omega}^{S} \pi R^{\alpha+2}}{(\alpha+2)}\left\{2(1+r)^{\alpha+2}-2(1-r)^{\alpha+2}\right\} .
$$

In the 2-D model, the term $\xi_{i}$ can be written as $\mathcal{H}_{i k}+\sum_{m=1}^{m=6} \mathcal{H}_{i m}$. Thus, we can rewrite $\Omega_{3}=\hat{\omega}^{N}$ $\sum_{i \in N_{k}}\left\{1+\sum_{m=1}^{m=6}\left(\mathcal{H}_{i m} / \mathcal{H}_{i k}\right)\right\}$ and $\Omega_{4}=\hat{\omega}^{S} \sum_{i \in S_{k}}\{1+$ $\left.\sum_{m=1}^{m=6}\left(\mathcal{H}_{i m} / \mathcal{H}_{i k}\right)\right\}$, and by symmetry of the cell model, we can further write $\Omega_{3}=\hat{\omega}^{N}\left\{\sum_{i \in N_{k}} 1+6 \sum_{i \in N_{k}}\left(\mathcal{H}_{i 1} / \mathcal{H}_{i k}\right)\right\}$ and $\Omega_{4}=\hat{\omega}^{S}\left\{\sum_{i \in S_{k}} 1+6 \sum_{i \in S_{k}}\left(\mathcal{H}_{i 1} / \mathcal{H}_{i k}\right)\right\}$.

Now, given $d N=\eta d S$, we can easily write $\Omega_{3}=$ $\hat{\omega}^{N} \eta\left\{\pi R^{2}(1-r)^{2}+6 \iint_{N_{k}}\left(\rho / \rho_{1}\right)^{\alpha} d S\right\}$ and $\Omega_{4}=$ $\hat{\omega}^{S} \eta\left\{4 \pi R^{2} r+6 \iint_{S_{k}}\left(\rho / \rho_{1}\right)^{\alpha} d S\right\}$, where $\rho_{1}$ is the distance between $d S$ and $\mathrm{AN}_{1}$. Note that the distance between $\mathrm{AN}_{k}$ and $\mathrm{AN}_{1}$ is $(\pi / \sqrt{3})^{(1 / 2)} R$. Thus, the distance $\rho_{1}$ as a function of $\rho$ and $\theta$ is $\left(\rho^{2}-2(\pi / \sqrt{3})^{(1 / 2)} R \rho \sin \theta+(\pi / \sqrt{3}) R^{2}\right)^{(1 / 2)}$.

Now, let $s=\rho / R$, where $0 \leq s \leq 1-r$ and $1-r \leq s \leq$ $1+r$, respectively, for $N_{k}$ and $S_{k}$. Let $t=\theta / \pi$, where $0 \leq$ $t \leq 2$ for both $N_{k}$ and $S_{k}$. By a simple calculation, we obtain $\left(\rho / \rho_{1}\right)^{\alpha}=\left(s^{\alpha} /\left(s^{2}-2(\pi / \sqrt{3})^{1 / 2} s \sin (\pi t)+(\pi / \sqrt{3})\right)^{\alpha / 2} \equiv\right.$ $f_{\alpha}(s, t)$ and, therefore

$$
\Omega_{3}=\eta \hat{\omega}^{N} \pi R^{2}\left\{(1-r)^{2}+6 \int_{s=0}^{s=1-r} \int_{t=0}^{t=2} s f_{\alpha}(s, t) d s d t\right\}
$$

and

$$
\Omega_{4}=\eta \hat{\omega}^{S} \pi R^{2}\left\{4 r+6 \int_{s=1-r}^{s=1+r} \int_{t=0}^{t=2} s f_{\alpha}(s, t) d s d t\right\} .
$$

Relation (8) is simply obtained by replacing $\Omega_{1}, \Omega_{2}, \Omega_{3}$, and $\Omega_{4}$ by their expressions in (10).

\section{B. Linear Model}

In the linear model (see Fig. 9), the width of cells is considered negligible as compared with the length. Therefore, the density $\eta$ represents a linear density. In other words, by letting $d u$ be an element of length, the density $\eta$ can be expressed as $d N / d u$; where again $d N$ is the number of MNs in the element $d u$. The variable $u$ belongs to $[-(L-a), L-a]$ and $[-(L+a),-(L-a)] \cup[L-a, L+a]$, respectively, for $N_{k}$ and 
$S_{k}$. By performing the change of variable, $l=u / L$, and given that $r=a / L$, we can derive the following

$$
\Omega_{1}=\frac{\eta \hat{\omega}^{N} L^{\alpha+1}}{(\alpha+1)}\left\{2(1-r)^{\alpha+1}\right\}
$$

and

$$
\Omega_{2}=\frac{\eta \hat{\omega}^{S} L^{\alpha+1}}{(\alpha+1)}\left\{2(1+r)^{\alpha+1}-2(1-r)^{\alpha+1}\right\} .
$$

Since in the linear model, the term $\xi_{i}$ evaluates as $\xi_{i}=\mathcal{H}_{i k}+$ $\mathcal{H}_{i 1}+\mathcal{H}_{i 2}$, we can rewrite

$$
\Omega_{3}=\eta \hat{\omega}^{N} L\left\{2(1-r)+2 \int_{0}^{1-r} \frac{(2-l)^{-\alpha}+(2+l)^{-\alpha}}{l^{-\alpha}} d l\right\}
$$

and

$$
\Omega_{4}=\eta \hat{\omega}^{S} L\left\{4 r+2 \int_{1-r}^{1+r} \frac{(2-l)^{-\alpha}+(2+l)^{-\alpha}}{l^{-\alpha}} d l\right\} .
$$

Relation (9) is simply obtained by replacing $\Omega_{1}, \Omega_{2}, \Omega_{3}$, and $\Omega_{4}$ by their expressions in (10).

\section{ACKNOWLEDGMENT}

The authors would like to thank the ITSUMO project members at Telcordia Technologies for initially suggesting the problem. The authors also wish to thank V. Phipatanasuphorn for the implementation of SHIP.

\section{REFERENCES}

[1] C.-C. Lee and R. Steele, "Effect of soft and softer handoffs on CDMA system capacity," IEEE Trans. Veh. Technol., vol. 47, pp. 830-841, Aug. 1998.

[2] Y. B. Lin and A. C. Pang, "Comparing soft and hard handoffs," IEEE Trans. Veh. Technol., vol. 49, pp. 792-798, May 2000.

[3] D. Tcha, S. Kang, and G. Jin, "Load analysis of the soft handoff scheme in a CDMA cellular system," IEEE J. Select. Areas Commun., vol. 19, pp. 1147-1152, June 2001.

[4] W. Choi and J. Kim, "Forward-link capacity of a DS/CDMA system with mixed multirate sources," IEEE Trans. Veh. Technol., vol. 50, no. 3, pp. 737-749, May 2001.

[5] R. Uc and R. Lara, "Forward link capacity losses for soft and softer handoff in cellular systems," in Proc. IEEE Int. Symp. Personal Indoor Mobile Radio Communications (PIMRC), vol. 1, 2001, pp. D48-D53.

[6] F. Ling, R. Love, M. Wang, T. Brown, P. Fleming, and H. Xu, "Behavior and performance of power controlled IS-95 reverse-link under soft handoff," IEEE Trans. Veh. Technol., vol. 49, pp. 1697-1704, Sept. 2000 .

[7] I. Vukovic, R. Pazhyannur, I. Ali, and P. Fleming, "Reordering soft handoff frames to minimize delay in CDMA cellular networks," in Proc. IEEE Int. Conf. Communications, vol. 5, 2002, pp. 3227-3233.

[8] A. Aksu, W. Biagini, and H. Stellakis, "Forward link soft-handoff gain of IS-95 CDMA system for wireless local loop networks," in Proc. IEEE Int. Symp. Personal Indoor Mobile Radio Communications (PIMRC), vol. 1, 1998, pp. 6-10.

[9] H. Fu and J. S. Thompson, "Downlink capacity analysis in 3 GPP WCDMA networks system," in Proc. Inst. Elect. Eng. Conf. Publication, 2002, pp. 534-538.
[10] A. El Gamal and T. Cover, "Achievable rates for multiple descriptions," IEEE Trans. Inform. Theory, vol. 28, pp. 851-857, Nov. 1982.

[11] M. Alasti, K. Sayrafian-Pour, and A. Ephremides, "Multiple description coding in networks with congestion problem," IEEE Trans. Inform. Theory, vol. 47, pp. 891-902, Mar. 2001.

[12] J. Ridge, F. Ware, and J. Gibson, "Permuted smoothed descriptions and refinement coding for mages," IEEE J. Select. Areas Commun., vol. 18, pp. 915-926, June 2000.

[13] W. W. Peterson and E. J. Weldon, Error-Correcting Codes, 2nd ed: The Massachusetts Institute of Technology, 1990.

[14] J. Kwon and D. Sung, "Soft handoff modelig in CDMA cellular systems," in Proc. 47th IEEE Vehicular Technology Conf., vol. 3, May 1997, pp. $1548-1551$.

[15] M. Guardiola and V. Rosales, "CDMA soft handoff modeling: a networking approach," in Proc. 48th IEEE Vehicular Technology Conf., vol. 2, May 1998, pp. 1641-1645.

[16] B. H. Cheung and V. C. M. Leung, "Network configurations for seamless support of CDMA soft handoffs between cell-clusters," IEEE J. Select. Areas Commun., vol. 15, pp. 295-299, Sept. 1997.

[17] F. Casado and H. M. Al-Housami, "Calculation of soft handoff gain for UMTS," in Proc. Inst. Elect. Eng. Conf.-Pub., 2002, pp. 42-46.

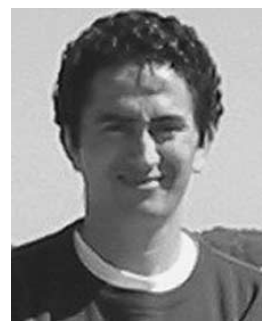

Bechir Hamdaoui received the B.S. degrees in both electrical and mechanical engineering, the M.S. degree in mechanical engineering from the National School of Engineering, Tunis (BAC+6+DEA, ENIT), Tunisia, in 1997 and 1998, respectively, and the M.S. degree in electrical and computer engineering from the University of Wisconsin, Madison, WI, in 2002, where he is currently working toward the Ph.D. degree.

From 1998 to 1999 , he worked as a quality control and planning engineer on power generation plant project under the supervision of FIAT Avio. His research focuses on various aspects in the area of computer networking including mobile networks, wireless communication systems, and ad hoc networks.

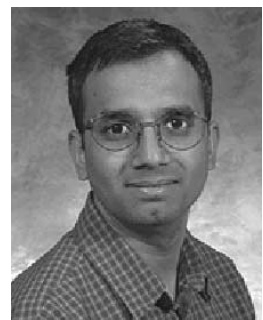

Parameswaran Ramanathan received the B.Tech. degree from the Indian Institute of Technology, Bombay, India, in 1984, and the M.S.E. and Ph.D. degrees from the University of Michigan, Ann Arbor, in 1986 and 1989, respectively.

Since 1989, he has been faculty member in the Department of Electrical and Computer Engineering, University of Wisconsin, Madison, where he is presently a Full Professor. He leads research projects in the areas of sensor netwoks and next-generation cellular technology. From 1997 to 1998 , he took a sabbatical leave to visit research groups at AT\&T Laboratories and Telcordia Technologies. His research interests include wireless and wireline networking, real-time systems, fault-tolerant computing, and distributed systems.

Dr. Ramanathan is presently an Associate Editor of the IEEE TRANSACTIONS ON MoBILE COMPUTING and Elsevier AdHoc Networks Journal. He served as an Associate Editor of IEEE TRANSACTIONS ON PARALLEL AND DISTRIBUTED COMPUTING from 1996 to 1999 . He has also served on program committees of conferences such as MOBICOM, Mobihoc, International Conferences on Distributed Systems and Networks, Distributed Computing Ssytems, Fault-Tolerant Computing Symposium, Real-time Systems Symposium, Conference on Local Computer Networks, and the International Conference on Engineering Complex Computer Systems. He was the Finance and Registration Chair for the 1999 Fault-Tolerant Computing Symposium. He was the Program Chairman of the Workshop on Architectures for Real-Time Applications in 1994 and the Program Vice-Chair for the International Workshop on Parallel and Distributed Real-Time Systems in 1996. He is a member of Association of Computing Machinery. 